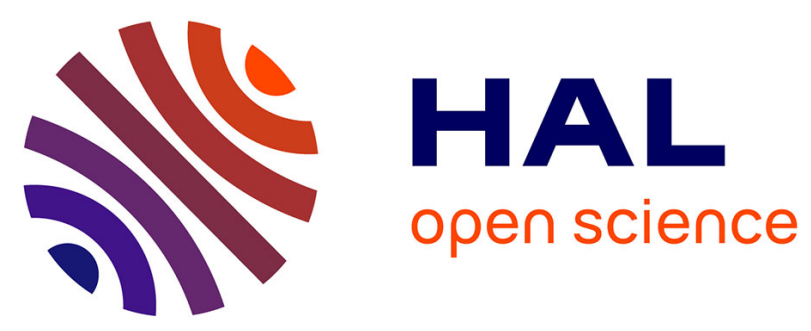

\title{
Influence of magnetic domain size on dipolar interactions and hysteresis field asymmetry in layered high/low coercivity perpendicular anisotropy systems
}

Thomas Hauet, Olav Hellwig

\section{- To cite this version:}

Thomas Hauet, Olav Hellwig. Influence of magnetic domain size on dipolar interactions and hysteresis field asymmetry in layered high/low coercivity perpendicular anisotropy systems. Journal of Applied Physics, 2014, 115 (12), 10.1063/1.4870136 . hal-01282857

\section{HAL Id: hal-01282857 https://hal.science/hal-01282857}

Submitted on 16 Aug 2016

HAL is a multi-disciplinary open access archive for the deposit and dissemination of scientific research documents, whether they are published or not. The documents may come from teaching and research institutions in France or abroad, or from public or private research centers.
L'archive ouverte pluridisciplinaire HAL, est destinée au dépôt et à la diffusion de documents scientifiques de niveau recherche, publiés ou non, émanant des établissements d'enseignement et de recherche français ou étrangers, des laboratoires publics ou privés. 


\title{
Influence of magnetic domain size on dipolar interactions and hysteresis field asymmetry in layered high/low coercivity perpendicular anisotropy systems
}

\author{
Thomas Hauet $^{1,2}$ and Olav Hellwig ${ }^{1}$ \\ 1. San Jose Research Center, HGST, a Western Digital Company, 3403 Yerba Buena Road, San Jose, CA 95135 \\ 2. Institut Jean Lamour, UMR CNRS 7198, Université de Lorraine, 54506 Vandoeuvre lès Nancy, France
}

\begin{abstract}
Dipolar interactions in low-coercivity/Pd/high-coercivity $[\mathrm{Co} / \mathrm{Pd}]_{\mathrm{X}} / \mathrm{Pd} /[\mathrm{Co} / \mathrm{Pd}]_{10}$ multilayer systems are studied as a function of the domain size in the $[\mathrm{Co} / \mathrm{Pd}]_{\mathrm{X}}$ low-coercivity layer $(\mathrm{LCL})$, while maintaining the domain size in the high-coercivity layer (HCL) constant. As the number of repeats $\mathrm{X}$ increases from 5 to 30 , the mean domain size of $[\mathrm{Co} / \mathrm{Pd}]_{\mathrm{X}} \mathrm{LCL}$ decreases from hundreds of micron to hundreds of nanometers. After demagnetization, different regimes can be distinguished from partial or exact domain duplication for LCL domain size larger than HCL one, to uncorrelated behavior for much smaller LCL domains. The results observed via magnetic force microscopy allow understanding the symmetry and asymmetry versus externally applied field as observed in macroscopic magnetometry measurements.
\end{abstract}

\section{INTRODUCTION}

A new interest has grown the last few years in antiferromagnetically coupled multilayers or in soft/hard bilayers with out-of-plane anisotropy due to their technological potential for AFC media [1], domain wall assisted recording [2] or multilevel recording applications $[3,4]$. In such systems, as soon as the magnetic layers are in a multi-domain state or as the lateral size of the sample is reduced, the short range exchange coupling, positive or negative, between successive layers competes with long range dipolar interactions. In the past we have used this competition to mimic the temperature dependent antiferromagnetic-ferromagnetic transition of $\mathrm{FeRh}$ with a simple perpendicular AF-coupled system [5]. In this multilayer, as well as more generally in all heterogeneous multilayers like in a hard/soft bilayer system, the intrinsic magnetic properties, e.g. exchange length, of each layer are different. As a consequence, the dipolar interaction generally acts between layers with different mean domain sizes or shapes. However, so far, no work has been devoted to systematically study the influence of the relative domain size on the dipolar interaction in out-ofplane multilayers.

$[\mathrm{Co} / \mathrm{Pd}]_{\mathrm{X}}$ multilayers are a suitable model system for such a study since in such systems the magnetic properties can be controlled via the thickness of each layer and the number of multilayer repeats $X$ [1] The perpendicular anisotropy of the $\mathrm{Co} / \mathrm{Pd}$ system originates from the Co surface anisotropy and the Co layers show strong ferromagnetic coupling across the $\mathrm{Pd}$ layers, so that a $[\mathrm{Co} / \mathrm{Pd}]_{\mathrm{X}}$ multilayer can be viewed as a single ferromagnetic (FM) layer with an average perpendicular anisotropy. Here we present a study of the dipolar interaction in a low-coercivity/Pd/high-coercivity out-of-plane anisotropy multilayer stack, namely $[\mathrm{Co} / \mathrm{Pd}]_{\mathrm{X}} / \mathrm{Pd}(10 \mathrm{~nm}) /[\mathrm{Co} / \mathrm{Pd}]_{10}$, as a function of the relative domain size in both magnetic layers. The thick $\operatorname{Pd}(10 \mathrm{~nm})$ spacer prevents any direct exchange coupling between the two ferromagnetic units, but only slightly decreases the inter-layer dipolar interactions. The mean domain size within the low-coercivity layer (LCL) is tuned in varying the number of repeats $\mathrm{X}$, while the highcoercivity layer (HCL) domain size is kept constant from one sample to another. Magnetic force microscopy (MFM) images are shown on samples with $5 \leq \mathrm{X} \leq 30$ after demagnetization to distinguish the different domain size regimes with varying $\mathrm{X}$. Combining MFM and magnetometry measurements, we explain the observed symmetry features of the LCL reversal process as a function of external magnetic field for the case of a nonuniform HCL domain state.

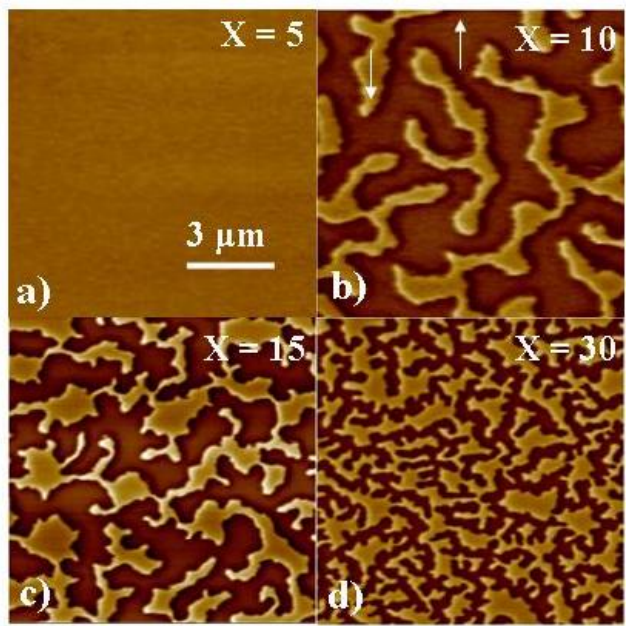

Figure 1. $10 * 10 \mu \mathrm{m}^{2}$ MFM images of demagnetized $[\mathrm{Co} / \mathrm{Pd}]_{\mathrm{X}}$ grown in a 3mTorr Ar pressure with $\mathrm{X}=\mathbf{5}(\mathrm{a}), 10$ (b), 15 (c), 30 (d) respectively. The up and down arrows represent the up and down out-of-plane magnetization states respectively.

\section{EXPERIMENTAL METHODS}

Using DC magnetron sputtering, $\mathrm{Pd}(3 \mathrm{~nm}) /$ $[\mathrm{Co}(0.3 \mathrm{~nm}) / \mathrm{Pd}(0.8 \mathrm{~nm})]_{\mathrm{X}} / \mathrm{Pd}(1.2 \mathrm{~nm})$ and $\mathrm{LCL} / \mathrm{Pd} / \mathrm{HCL}$ $\mathrm{Pd}(3 \mathrm{~nm}) / \quad[\mathrm{Co}(0.3 \mathrm{~nm}) / \mathrm{Pd}(0.8 \mathrm{~nm})]_{\mathrm{X}} / \quad \operatorname{Pd}(10 \mathrm{~nm}) /$ $[\mathrm{Co}(0.3 \mathrm{~nm}) / \mathrm{Pd}(0.8 \mathrm{~nm})]_{10} / \mathrm{Pd}(1.2 \mathrm{~nm})$ multilayers have been deposited onto $\mathrm{Si}$ wafers. The low coercivity $[\mathrm{Co} / \mathrm{Pd}]_{\mathrm{X}}$ multilayer has been deposited at 3mTorr Argon pressure and the high coercivity $[\mathrm{Co} / \mathrm{Pd}]_{10}$ multilayer at 7 
mTorr Argon pressure.[6] Various single and high/low coercivity bilayer samples have been grown with $5 \leq \mathrm{X} \leq$ 30. The macroscopic magnetic properties were measured using a commercial Vibrating Sample Magnetometry (VSM). Images of the magnetic domain configurations were obtained using Magnetic Force Microscopy (MFM). All measurements have been performed at room temperature.

\section{RESULTS AND DISCUSSION}

After AC demagnetization in a perpendicular field, a systematic MFM study of the single $[\mathrm{Co} / \mathrm{Pd}]_{X}$ multilayer domain size and shape is presented in Fig. 1. We find that domains are hardly stabilized in the thinnest sample containing five repeats. Indeed, as observed in previous studies [1], for $X=5$, the demagnetization field induces large micrometer size domains. As $\mathrm{X}$ increases, irregular two-dimensional domains form, whose mean domain width decreases to $900 \mathrm{~nm}, 700 \mathrm{~nm}$ and, $400 \mathrm{~nm}$ for $\mathrm{X}=10,15$ and 30 respectively. The bright domains, resp. dark domains, correspond to a magnetization pointing towards the negative, resp. positive, field direction perpendicular to the sample plane (called in the following "down", resp. "up"). The irregular pattern is typical of thin $\mathrm{Co} / \mathrm{Pd}$ films after AC-demagnetization in an out-of plane field [1]. To characterize the dipolar interaction between magnetic layers with different mean domain size, LCL/Pd/HCL multilayers have been fabricated, where the LCL consists of a $[\mathrm{Co} / \mathrm{Pd}]_{\mathrm{X}}$ multilayer deposited at $3 \mathrm{mTorr}$ and the HCL is a $[\mathrm{Co} / \mathrm{Pd}]_{10}$ multilayer deposited at a 7 mTorr argon pressure. The inset of Fig. 2 shows a MFM image of the domain structure obtained after the same demagnetization procedure in the case of the high-Hc $[\mathrm{Co} / \mathrm{Pd}]_{10}$ multilayer. The mean domain size $(1.2 \mu \mathrm{m})$ is similar to the one measured for the same number of repeats grown at 3 mTorr, but the dispersion in domain size and shape is much more pronounced. This difference is due to the increase of the multilayer interface roughness and crystallographic defects induced by the higher deposition pressure [6]. These defects usually lead as well to a higher coercivity and the magnetization reversal process of the high pressure film is then dominated by more frequent domain nucleation processes.

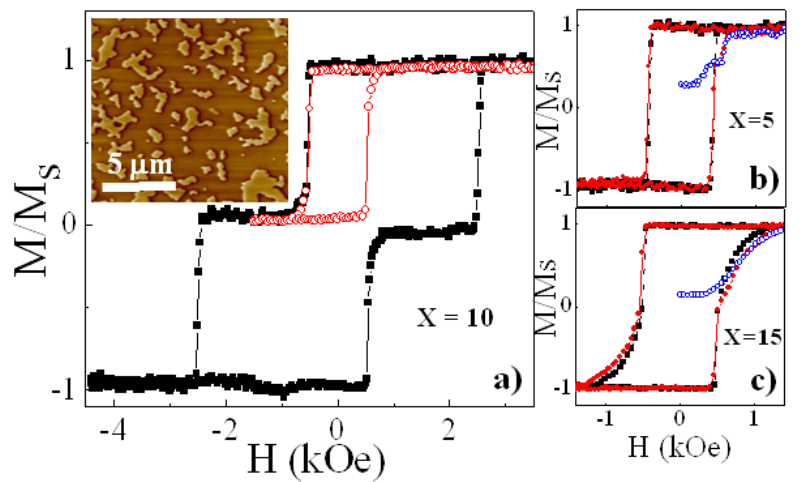

Figure 2: a) Normalized hysteresis loops of LCL/Pd/HCL $[\mathrm{Co} / \mathrm{Pd}]_{10} / \mathrm{Pd} /[\mathrm{Co} / \mathrm{Pd}]_{10}$ measured at $300 \mathrm{~K}$. Square solid symbols show the major loop, while open circles correspond to a minor loop with the HCL in a uniform state. Inset: $25 * 25 \mu^{2}$ MFM image of demagnetized $[\mathrm{Co} / \mathrm{Pd}]_{10}$ sample grown at $7 \mathrm{mTorr}$ Ar pressure. b,c) Normalized curve of first magnetization (open blue circle) and minor loop (red circle) measured after demagnetization of $[\mathrm{Co} / \mathrm{Pd}]_{\mathrm{X}} / \mathrm{Pd} /[\mathrm{Co} / \mathrm{Pd}]_{10}$ compared with the minor loop obtained in the case of uniformly magnetized HCL (black symbol and dash line)

Fig. 2a presents hysteresis loops measured on a $\mathrm{LCL} / \mathrm{Pd} / \mathrm{HCL}[\mathrm{Co} / \mathrm{Pd}]_{10} / \mathrm{Pd} /[\mathrm{Co} / \mathrm{Pd}]_{10}$ multilayer at $300 \mathrm{~K}$ with the field perpendicular to the sample plane. The major loop reveals two distinct steps corresponding to the independent reversal of the LCL and the HCL. Starting from positive saturation the LCL magnetization reverses at $\mathrm{H}_{\mathrm{N}}^{0}=-500$ Oe and stays anti-parallel to the HCL $[\mathrm{Co} / \mathrm{Pd}]_{10}$ until about $\mathrm{H}=-2.5 \mathrm{kOe}$, where the $\mathrm{HCL}$ magnetization reversal occurs. The major loop is essentially the same independent of the number of $\mathrm{Co} / \mathrm{Pd}$ repeats in the LCL. The coercivity of the HCL increases slightly as $\mathrm{X}$ increases, most probably because of an increase of the roughness on top of the bottom LCL. A minor field cycle performed between $+/-1.5 \mathrm{kOe}$ shows no residual bias field and indicates that the $\mathrm{Pd}(10 \mathrm{~nm})$ layer in between the two ferromagnetic multilayers prevents any direct exchange interaction (Fig. 2).

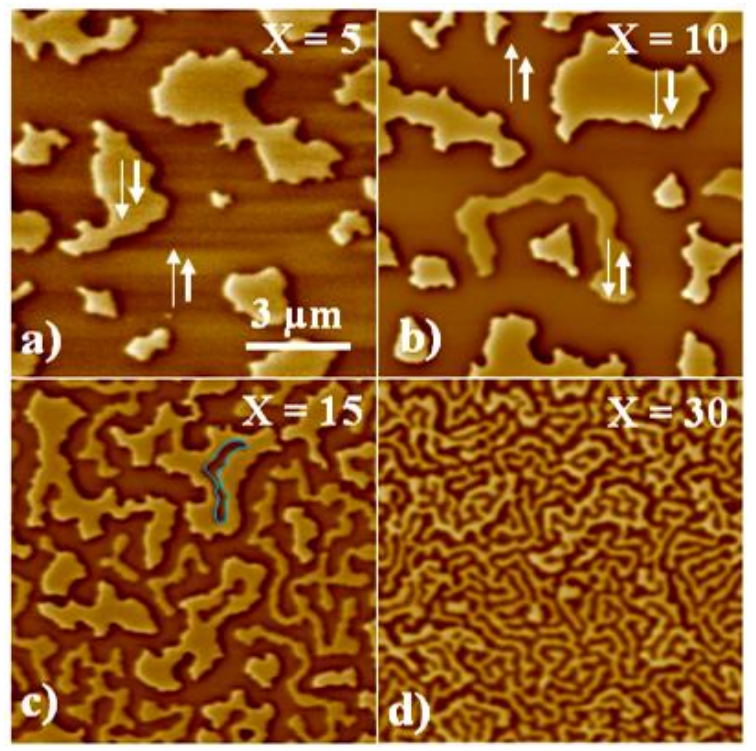

Figure 3. $10 * 10 \mu \mathrm{m}^{2}$ MFM images of demagnetized LCL/Pd/HCL $[\mathrm{Co} / \mathrm{Pd}]_{\mathrm{X}} / \mathrm{Pd} /[\mathrm{Co} / \mathrm{Pd}]_{10}$ with $\mathrm{X}=5(\mathrm{a}), 10$ (b), 15 (c), 30 (d) respectively. The short and long arrows represent out-of-plane magnetization states of the HCL and $L C L$ respectively. The blue contour in (c) corresponds to the boundaries of a specific domain described in the text.

Fig. 3 presents a series of MFM images of the demagnetized state of the $[\mathrm{Co} / \mathrm{Pd}]_{\mathrm{X}} / \mathrm{Pd} /[\mathrm{Co} / \mathrm{Pd}]_{10}$ bilayer films with varying values of repeats $X=5,10,15$ and 30 . The samples have been demagnetized using the same procedure as that described for the single layer films. Using a Pd spacer thickness $(10 \mathrm{~nm})$, the MFM sensibility allows us to probe the stray field resulting from the top HCL combined with that from the bottom LCL. In the 
case of the thickest LCL, the MFM was sensitive only to the first top few nanometers of the LCL; however, as discussed above, $[\mathrm{Co} / \mathrm{Pd}]_{\mathrm{X}}$ can be viewed as a single ferromagnetic (FM) layer and no variation of the magnetic domain structure is expected in depth of the layer. In Fig. 3a, showing the $X=5$ case, the image displays mostly dark and bright domains. There is no domain with a distinguishable intermediate contrast in the image. The dark and bright contrast observed in Fig. 3a may respectively correspond to configurations where the "bottom LCL - top HCL" system is saturated "up-up" or "down-down", but it may also correspond to a zone of uniform LCL magnetization with up and down HCL domains. Unfortunately, it is not possible from the MFM image alone to conclude this. Nevertheless, when measuring the curve of first magnetization after demagnetization, a two step curve is measured and the saturation occurs at a 650 Oe field, i.e. 150 Oe higher than the saturation field of the LCL when the HCL magnetization is uniform (Fig. 2b). Similar observations, related to the effect of domain duplication, have already been reported previously in Ref. [7] and [8]. Such an effect is corroborated by the fact that $\operatorname{Pd}(10 \mathrm{~nm})$ is thin enough to allow a strong dipolar coupling [9], so we can expect that exact replication must occur as soon as the LCL and HCL domain sizes are of the same order. An element specific technique and a sample including for instance $\mathrm{Ni}$ in one of the two layers could help measuring the LCL domain size range, where perfect domain replication occurs. Interestingly, the minor loop performed with a demagnetized HCL shows a kink only on the ascending branch (red curve in Fig. 2(b)). This asymmetry, as well as the LCL and HCL domain superposition in Fig. 3(a), is discussed in more detail hereafter. Similar MFM images and magnetization loops are observed for $\mathrm{X}$ up to 8 .

In Fig. 3b, in $[\mathrm{Co} / \mathrm{Pd}]_{10} / \mathrm{Pd} /[\mathrm{Co} / \mathrm{Pd}]_{10}$, a third type of domain appears on MFM images that is characterized by an intermediate darkness and the absence of a black inner contour. The black inner boundary is seen on most of the bright domains on Fig. $3 a$ and $3 b$, and originates from the stray field profile whose intensity maximum is localized at the boundary between domains of opposite magnetization. The previous domain features, as well as its shape and size in comparison with Fig.2b, indicates that this new "down" domain lays in the LCL while the HCL magnetization is kept in the opposite direction, i.e. "up". This new state is defined as "down-up" state. The fact that this new domain in Fig.3b spreads around the brighter domains is a confirmation that the "down-down" domains belong to both HCL and LCL. Indeed if there was no domains inside the LCL, the most energetically stable position for the new LCL domain, would be on top of the HCL domains [6]. From $X=8$ to $X=15$, the density of LCL domains increases since the mean domain size decreases (as explained above).

For $\mathrm{X}$ equal to or higher than 15 , as shown in Fig. 3c, the demagnetized state contains four different contrast levels. In addition to the brightest "down-down" domains and the darkest "up-up" domains, intermediate contrast "down-up" domains spread like a de-wetting fluid inside the dark up-up domains, while avoiding the proximity of any "up-up"/"down-down" domain walls. Similarly "up-down" domains of dark contrast appear inside the brightest domains. At this stage intrinsic domain sizes in the HCL and LCL have become very different and thus are incompatible with each other. Underneath a "down" HCL domain, the LCL domains lay primarily where the dipolar field intensity is the strongest, i.e. close to the boundary of the HCL domains. But the self demagnetization field inside the LCL is strong enough to create an "up" domain in the center of the HCL domain (e.g. blue contour in Fig. 3c). This result reveals the competition between dipolar fields from the HCL domain and the self demagnetization field inside the LCL, whose relative influences vary as $\mathrm{X}$ increases.
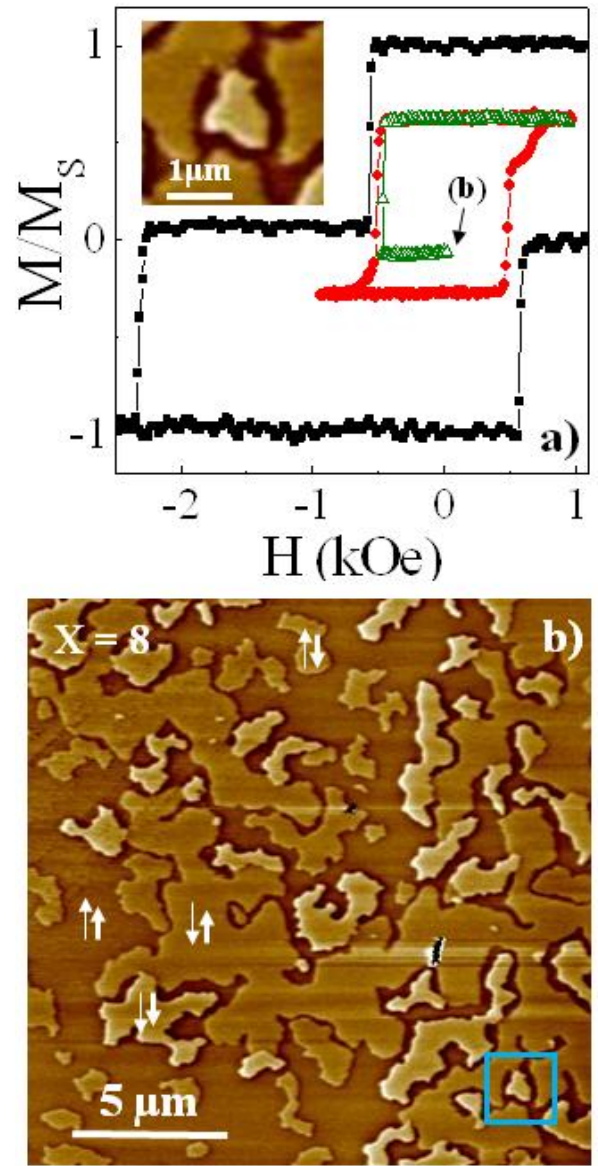

Figure 4. a) Normalized hysteresis loops of $L C L / P d / H C L$ $[\mathrm{Co} / \mathrm{Pd}]_{8} / \mathrm{Pd} /[\mathrm{Co} / \mathrm{Pd}]_{10}$ measured at $300 \mathrm{~K}$. Square solid black symbols show the major loop, while red solid circles correspond to a minor loop with the HCL in a demagnetized state. Partial LCL reversal (green triangles) within a minor loop (red circle) measured after demagnetization. Inset: zoom $\left(2.5 * 2.5 \mu \mathrm{m}^{2}\right)$ of the blue squared surface in the MFM image in b). b) $20 * 20 \mu \mathrm{m}^{2}$ MFM image of the remanent magnetic state after the partial LCL reversal (green triangle in a)). The short and long arrows represent out-of-plane magnetization states of the HCL and LCL respectively.

For $X$ larger than 15 , e.g. $X=30$ shown in Fig. $3 \mathrm{~d}$, the dipolar coupling between the LCL and HCL 
relative to the inner LCL demagnetization fields decreases. The HCL configuration now only locally constrains the shape and the location of the domains but not their width. The progressive decrease of the relative influence of the LCL/HCL dipolar interaction is also emphasized at the macroscopic scale by the progressive disappearance of the asymmetric kink in the minor hysteresis loop of $[\mathrm{Co} / \mathrm{Pd}]_{\mathrm{X}} / \mathrm{Pd} /[\mathrm{Co} / \mathrm{Pd}]_{10}$. In the $\mathrm{X}=30$ case (not shown here), the minor loops measured with and without domains in the HCL are identical. In the case of $X=15$, the curves shape are similar but the saturation field is still slightly higher in presence of HCL domains (Fig.2c). Note that for $X=15$, the difference between the minor loops is symmetric in field on the contrary to the $\mathrm{X}=5$ case (Fig. $2 \mathrm{~b}$ and $2 \mathrm{c}$ ).

To achieve a microscopic understanding of the asymmetry features of the minor loop obtained for low $\mathrm{X}$ values, a $\mathrm{LCL} / \mathrm{Pd} / \mathrm{HCL}[\mathrm{Co} / \mathrm{Pd}]_{8} / \mathrm{Pd} /[\mathrm{Co} / \mathrm{Pd}]_{10}$ multilayer has been first demagnetized to nucleate some domains in the HCL. Then the LCL has been saturated and partially reversed in a negative field (Fig.4a) in order to force a larger "down" domain surface in the LCL than in the HCL. Then, at remanence, the observed MFM image (Fig.4b) shows four levels of contrast. The brightest domains have the typical non-labyrinth shape of the HCL domains and correspond to "down-down" domains. From these "down-down" regions (see inset Fig. 2), "down-up" regions spread widely. These "down-up" domains grow over the whole layer except a band of about 300nm (in average) away from the "down-down" region boundaries.

Let's try to explain the origin of this extended pinning site. Fig. 5(a) presents a 1D scheme characteristic of this state. Fig. 5(c) represents the z-component of dipolar field originating from the HCL and applied onto the LCL. In the case of a single domain wall (DW) in a infinite film of thickness $h$, an effective DW width $\delta$, and a saturation magnetization $M \mathrm{~s}$, the dipolar stray field $\mathrm{H}_{z}$ perpendicular to the sample surface at a distance $z$ over the surface is given by Ref. [10,11] :

We used this expression to calculate the stray field coming from a 1D down magnetized HCL domain of 1 micron width as the stray field induced by two opposite DWs separated by 1 micron. We used $\mathrm{Ms}=550 \mathrm{emu} / \mathrm{cm}^{3}$, $\mathrm{h}=11 \mathrm{~nm}$ HCL thickness, $\delta=20 \mathrm{~nm}, \mathrm{z}=14.4 \mathrm{~nm}$ the distance between the HCL surface and the middle of the LCL stack. As already well documented in the literature $[11,12]$, an up field is created just outside the HCL down domain and a down dipolar field occurs over the whole HCL down domain. At that stage in the descending branch of the green minor hysteresis loop in Fig. 4(a), domain walls are pinned by the external up dipolar field induced by the "down-down" domain $[11,12]$. To reach negative saturation, i.e. to reverse the two thin up LCL domains in Fig. 5(a), the external field has to overcome the up dipolar field of the HCL. Very near the down-up and up-down HCL domain wall centers (marked by the two dashed lines at $\mathrm{x}=-0.5$ and $+0.5 \mu \mathrm{m}$ in Fig.5(c), which also indicate the positions of two LCL domain walls in Fig.5(a)), the amplitude of HCL induced up dipolar field is about $280 \mathrm{Oe}$ and then it decreases to 60 Oe at $300 \mathrm{~nm}$ away from the HCL domain wall centers ( (marked by each dashed line at $\mathrm{x}=-0.8$ and $+0.8 \mu \mathrm{m}$ in Fig.5(c), which also indicate the position of two LCL domain walls in Fig.5(a) and (b)).
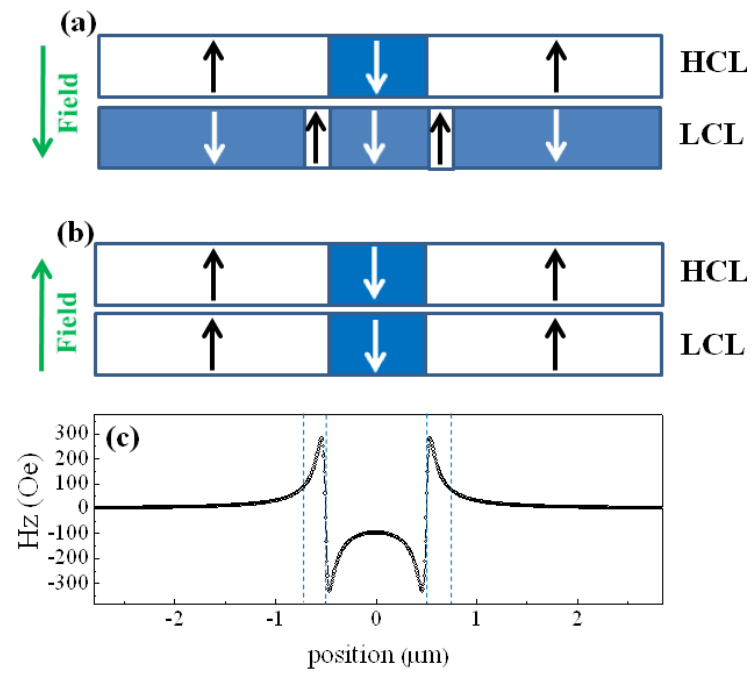

Figure 5: One-dimensional scheme of the magnetic domain configuration in the LCL/Pd/HCL bilayer system around a 1 micron wide HCL isolated down domain when field cycling the LCL within a minor loop. The LCL domain configuration is shown before reaching negative (a) and positive (b) saturation in an external magnetic field respectively. (c) One dimensional calculated stray fields originating from a $1 \mu \mathrm{m}$ wide HCL down magnetized domain, between 2 up magnetized domains. The dashed lines are guides for the eyes indicating the domain boundaries in (a) and (b).

During the ascending branch of the red minor loop in Fig. 4(a), the LCL reversal starts below the wide region of the HCL up domains and spreads until the LCL domain walls get pinned by the negative dipolar fields that are created by the HCL down domains. This state corresponds to the scheme in Fig. 5(b). Such a magnetic state is stabilized by the strong HCL dipolar field, whose amplitude is $330 \mathrm{Oe}$ at the edge and 100 Oe in the middle of the $1 \mu \mathrm{m}$ large domain as calculated in Fig. 5(c). The additional required field to achieve LCL saturation gives rise to the kink observed in the ascending branch of the red curve in Fig. 4(a). The asymmetry of the red curve in Fig. 4(a) reveals the asymmetry of the de-pinning process linked to the HCL magnetic configuration. The smaller are the HCL domains, the more asymmetric are the dipolar fields, therefore the more pronounced is the asymmetry observed in the hysteresis loop.

\section{CONCLUSION}

In summary, we have characterized the dipolar interaction in $\mathrm{LCL} / \mathrm{Pd} / \mathrm{HCL}$, namely $[\mathrm{Co} / \mathrm{Pd}]_{\mathrm{X}} / \mathrm{Pd} /$ $[\mathrm{Co} / \mathrm{Pd}]_{10}$ multilayer systems. Varying $\mathrm{X}$ allows changing the mean domain size of the LCL, while the HCL domain 
size is kept constant. When the LCL domain size is larger than HCL domain size, the domains contained in the HCL are exactly replicated in the LCL during demagnetization as well as LCL reversal, at least when the difference in domain sizes is not too large. As $\mathrm{X}$ increases and more precisely as soon as the LCL domain size is more or less three times lower than HCL domain size, the width of the LCL domains is no longer correlated to the HCL domains, but their location and shape are. Asymmetric loops have been measured and correlated to the asymmetry in the dipolar fields induced by HCL domains. Such MFM studies as presented here help providing a good understanding of the symmetries and asymmetries observed in hysteresis loops of dipolar coupled LCL/spacer/HCL perpendicular anisotropy magnetic bilayer systems.

\section{References}

[1] O. Hellwig, A. Berger, J.B. Kortright, Eric E. Fullerton, J. Magn. Magn. Mater. 319, 13 (2007)

[2] D. Suess, T. Schrefl, S. Fahler, M. Kirschner, G. Hrkac, F. Dorfbauer, and J. Fidler, Appl. Phys. Lett. 87, 012504 (2005).

[3] M. Albrecht, G. Hu, A. Moser, O. Hellwig, and B. D. Terris, J.Appl. Phys. 97, 103910 (2005).

[4] H. Awano, S. Imai, M. Sekine, M. Tani, N. Ohta, K. Mitani,

N. Takagi, H. Noguchi, and M. Kume, IEEE Trans. Magn. 36,

2261 (2000).

[5] J.-U. Thiele, T. Hauet, O. Hellwig, Appl. Phys. Lett. 92, 242502 (2008)

[Pierce2013] M. S. Pierce, J. E. Davies, J. J. Turner, K. Chesnel, E. E. Fullerton, J. Nam, R. Hailstone, S. D. Kevan, J. B.

Kortright, Kai Liu, L. B. Sorensen, B. R. York, and O. Hellwig, Phys. Rev. B 87 (2013) 184428.

[7] T. Hauet, C. Günther, B. Pfau, M. Schabes, J.-U. Thiele, R. L. Rick, P. Fischer, S. Eisebitt, and O. Hellwig, Phys. Rev. B 77, 184421 (2008)

[8] B. Rodmacq, V. Baltz, B. Dieny, Phys. Rev. B 73, 092405

(2006)

[9] V. Baltz, A. Marty, B. Rodmacq, B. Dieny, Phys. Rev. B 75, 014406 (2007)

[10] D. Rugar, H. J. Mamin, P. Guethner, S. E. Lambert, J. E. Stern, I. McFadyen, and T. Yogi. J. Appl. Phys. 68, 1169 (1990) [11] M. Gottwald, M. Hehn, D. Lacour, T. Hauet, F. Montaigne, S. Mangin, P. Fischer, M.-Y. Im, A. Berger, Phys. Rev. B 85, 064403 (2012)

[12] S. Wiebel, J.-P. Jamet, N. Vernier, A. Mougin, J. Ferre, V. Baltz, B. Rodmacq, and B. Dieny, Appl. Phys. Lett. 86, 142502 (2005) 